\title{
$\Delta$-convergence for proximal point algorithm and fixed point problem in CAT(0) spaces
}

Shamshad Husain ${ }^{1 *}$ (D) and Nisha Singh ${ }^{1}$

"Correspondence:

s_husain68@yahoo.com

${ }^{1}$ Department of Applied

Mathematics, Aligarh Muslim

University, Aligarh, India

\begin{abstract}
In this paper, we prove the $\Delta$-convergence of a modified proximal point algorithm for common fixed points in a CAT(0) space for different classes of generalized nonexpansive mappings including a total asymptotically nonexpansive mapping, a multivalued mapping, and a minimizer of a convex function. The results in this paper generalize the corresponding results given by some authors. Moreover, numerical example is given to illustrate and show the $\Delta$-convergence of the proposed algorithm for supporting our result.
\end{abstract}

MSC: 47H09; 47H10;47J25; 65K10

Keywords: Proximal point algorithm; Total asymptotically nonexpansive mapping; Nonexpansive multivalued mapping; $\Delta$-convergence

\section{Introduction}

Let $(Y, \theta)$ be a geodesic space and $h: Y \rightarrow(-\infty, \infty]$ be a proper convex function. By $\arg \min _{r \in Y} h(r)$, we denote the set of minimizers of a convex function. To solve $s \in Y$ such that

$$
h(s)=\min _{r \in Y} h(r)
$$

in optimization theory a powerful tool is the well-known PPA which was introduced by Martinet [1].

Rockafellar [2] studied, the convergence to a solution of the convex minimization problem in the framework of a Hilbert space by the PPA. He also proved that the sequence $\left\{s_{m}\right\}$ converges weakly to a minimizer of a convex function $h$ such that $\sum_{m=1}^{\infty} \pi_{m}=\infty$.

Bacak [3] introduced the PPA in a $\operatorname{CAT}(0)$ space $(Y, \theta)$ as follows:

$$
\left\{\begin{array}{l}
s_{1} \in Y, \\
s_{m+1}=\arg \min _{r \in Y}\left[h(r)+\frac{1}{2 \pi_{m}} \theta^{2}\left(r, s_{m}\right)\right], \quad \forall m \in \mathbb{N},
\end{array}\right.
$$

where $\pi_{m}>0, \forall m \in \mathbb{N}$, and he showed that, if $h$ has a minimizer and $\sum_{m=1}^{\infty} \pi_{m}=\infty$, then the sequence $\left\{s_{m}\right\} \Delta$-converges to its minimizer [4].

(c) The Author(s) 2019. This article is distributed under the terms of the Creative Commons Attribution 4.0 International License (http://creativecommons.org/licenses/by/4.0/), which permits unrestricted use, distribution, and reproduction in any medium, provided you give appropriate credit to the original author(s) and the source, provide a link to the Creative Commons license, and indicate if changes were made. 
Cholamjiak [5] modified the PPA in $\operatorname{CAT}(0)$ spaces $(Y, \theta)$ by using the Halpern method as follows:

$$
\left\{\begin{array}{l}
r_{m}=\arg \min _{r \in Y}\left[h(r)+\frac{1}{2 r} \theta^{2}\left(r, s_{m}\right)\right], \\
s_{m+1}=a_{m} u \oplus\left(1-a_{m}\right) r_{m}, \quad \forall m \in \mathbb{N},
\end{array}\right.
$$

where $r>0, \lim _{m \rightarrow \infty} a_{m}=0$, and $\sum_{m=1}^{\infty} a_{m}=\infty$. He proved that the sequence $\left\{s_{m}\right\}$ converges to its minimizer.

Cholamjiak et al. [6] modified the following PPA in CAT(0) spaces as follows:

$$
\left\{\begin{array}{l}
q_{m}=\arg \min _{r \in Y}\left[h(r)+\frac{1}{2 \pi_{m}} \theta^{2}\left(r, s_{m}\right)\right], \\
r_{m}=\left(1-b_{m}\right) s_{m} \oplus b_{m} \mathcal{T}_{1} q_{m}, \\
s_{m+1}=\left(1-a_{m} \mathcal{T}_{1} s_{m} \oplus a_{m} \mathcal{T}_{2} r_{m}, \quad \forall m \in \mathbb{N}\right.
\end{array}\right.
$$

and established some strong convergence theorems of the proposed algorithm to common fixed points of nonexpansive mappings and to minimizers of a convex function. Chang et al. [7] established some strong convergence theorems of the PPA to a common fixed point of asymptotically nonexpansive mappings and to minimizers of a convex function in $\operatorname{CAT}(0)$ spaces.

Kitkuan and Padcharoen [8] studied the iteration process in CAT(0) spaces as follows:

$$
\left\{\begin{array}{l}
p_{m}=\left(1-a_{m}\right) s_{m} \oplus a_{m} \mathcal{T}^{m} s_{m}, \\
r_{m}=\left(1-b_{m}\right) p_{m} \oplus b_{m} \mathcal{T}^{m} p_{m}, \\
s_{m+1}=\left(1-c_{m}\right) r_{m} \oplus c_{m} \mathcal{T}^{m} r_{m}, \quad \forall m \in \mathbb{N},
\end{array}\right.
$$

where $\left\{a_{m}\right\},\left\{b_{m}\right\}$, and $\left\{c_{m}\right\}$ are real sequences in $(0,1)$. They also proved some strong convergence theorems for generalized asymptotically quasi-nonexpansive mappings under certain conditions.

Markin [9] and Nadler [10] introduced the study of fixed points for multivalued contractions and nonexpansive mappings using the Hausdorff metric. Shimizu and Takahashi [11] proved the existence of fixed points for multivalued nonexpansive mappings in convex metric spaces, that is, every multivalued mapping $\mathcal{T}: Y \rightarrow \mathcal{C}(Y)$ has a fixed point in a bounded, complete, and uniformly convex metric space $(y, \theta)$, where $\mathcal{C}(Y)$ is the family of all compact subsets of $Y$.

Motivated and inspired by the above results, we propose the modified proximal point algorithm with the process for three asymptotically nonexpansive mappings and multivalued mappings in CAT(0) spaces. Under suitable conditions, we prove some convergence theorems of the proposed method. Further, we provide a numerical example to illustrate and show the efficiency of the proposed algorithm for supporting our main results.

\section{Preliminaries}

A metric space $(Y, \theta)$ is called a CAT space if it is geodesically connected and every geodesic triangle in $Y$ is at least as "thin" as its comparison triangle in the Euclidean plane. 
A subset $W$ of a CAT(0) space $Y$ is said to be convex if, for any $s, r \in W$, we have $[s, r] \subset$ $W$, where

$$
[s, r]:=\{t s \oplus(1-t) r: 0 \leq t \leq 1\}
$$

is the unique geodesic joining $s$ and $r$.

In this paper, we can write $(1-t) s \oplus t r$ for the unique point $q$ in the geodesic segment joining from $s$ to $r$ such that

$$
\theta(s, q)=t \theta(s, r), \theta(r, q)=(1-t) \theta(s, r),
$$

where $t \in[0,1]$.

Lemma 2.1 Let $Y$ be a geodesic space in a $C A T(0)$ space. For all $s, r, q \in Y$ and $t \in[0,1]$, we have

(i) $\theta^{2}((1-t) s \oplus t r, q) \leq(1-t) \theta^{2}(s, q)+t \theta^{2}(r, q)-t(1-t) \theta^{2}(s, r)$,

(ii) $\theta((1-t) s \oplus t r, q) \leq(1-t) \theta(s, q)+t \theta(r, q)$.

Definition 2.1 Let $\mathcal{T}: W \rightarrow W$ be a mapping.

(i) An element $s \in W$ is said to be a fixed point of $\mathcal{T}$ if $s=\mathcal{T} s$. Denote by $G(\mathcal{T})$ the set of fixed points of $\mathcal{T}$;

(ii) semi-compact if every bounded sequence $\left\{s_{m}\right\} \subset W$, satisfying $\theta\left(s_{m}, \mathcal{T} s_{m}\right) \rightarrow 0$ as $m \rightarrow \infty$, has a convergent subsequence;

(iii) nonexpansive if $\theta(\mathcal{T} s, \mathcal{T} r) \leq \theta(s, r)$ for any $s, r \in W$;

(iv) asymptotically nonexpansive if there exists a sequence $\left\{e_{m}\right\} \subset[0,+\infty)$ and $\lim _{m \rightarrow \infty} e_{m}=0$ such that

$$
\theta\left(\mathcal{T}^{m} s, \mathcal{T}^{m} r\right) \leq\left(1+e_{m}\right) \theta(s, r), \quad \forall s, r \in W, m \geq 1 ;
$$

(v) $\left(\left\{v_{m}\right\},\left\{\mu_{m}\right\}, \rho\right)$-total asymptotically nonexpansive, if there exist nonnegative sequences $\left\{v_{m}\right\},\left\{\mu_{m}\right\}$ with $\mu_{m} \rightarrow 0, v_{m} \rightarrow 0$ and a strictly increasing continuous function $\rho:[0,+\infty) \rightarrow[0,+\infty)$ with $\rho(0)=0$ such that

$$
\theta\left(\mathcal{T}^{m} s, \mathcal{T}^{m} r\right) \leq \theta(s, r)+v_{m} \rho(\theta(s, r))+\mu_{m}, \quad \forall s, r \in W, m \geq 1 ;
$$

(vi) uniformly $L$-Lipschitzian if there exists a constant $L>0$ such that

$$
\theta\left(\mathcal{T}^{m} s, \mathcal{T}^{m} r\right) \leq L \theta(s, r), \quad \forall s, r \in W, m \geq 1
$$

Definition 2.2 Let $\left\{s_{m}\right\}$ be a bounded sequence in a $\operatorname{CAT}(0)$ space $(Y, \theta)$. For any $s \in Y$, we put

$$
\hat{r}\left(s,\left\{s_{m}\right\}\right)=\lim _{m \rightarrow \infty} \sup \theta\left(s, s_{m}\right) .
$$

Then,

1. The asymptotic radius $\hat{r}\left(\left\{s_{m}\right\}\right)$ of $\left\{s_{m}\right\}$ is given by

$$
\hat{r}\left(\left\{s_{m}\right\}\right)=\inf \left\{\hat{r}\left(s,\left\{s_{m}\right\}\right): s \in Y\right] .
$$


2. The asymptotic center $\mathcal{A}\left(\left\{s_{m}\right\}\right)$ of $\left\{s_{m}\right\}$ is the set

$$
\mathcal{A}\left(\left\{s_{m}\right\}\right)=\left\{s \in Y: \hat{r}\left(s,\left\{s_{m}\right\}\right)=\hat{r}\left(\left\{s_{m}\right\}\right)\right\} .
$$

In a complete $\mathrm{CAT}(0)$ space, $\mathcal{A}\left(\left\{s_{m}\right\}\right)$ consists of exactly one point [6].

Definition 2.3 A sequence $\left\{s_{m}\right\}$ in a CAT(0) space $Y$ is said to be $\Delta$-convergent to a point $s \in Y$ if $s$ is the unique asymptotic center of $\left\{u_{m}\right\}$ for every subsequence $\left\{u_{m}\right\}$ of $\left\{s_{m}\right\}$. In this case, we write $\Delta \lim _{m \rightarrow \infty} s_{m}=s$ of $\left\{s_{m}\right\}$ and denote $\mathcal{W}_{\Delta}\left(s_{m}\right):=\bigcup\left\{\mathcal{A}\left(\left\{u_{m}\right\}\right)\right\}$, where the union is sum over all subsequences $\left\{u_{m}\right\}$ of $\left\{s_{m}\right\}$.

Lemma $2.2([6])$ If $\left\{s_{m}\right\}$ is a bounded sequence in a complete $C A T(0)$ space with $\mathcal{A}\left(\left\{s_{m}\right\}\right)=$ $\{s\},\left\{u_{m}\right\}$ is a subsequence of $\left\{s_{m}\right\}$ with $\mathcal{A}\left(\left\{u_{m}\right\}\right)=\{u\}$, and the sequence $\left\{\theta\left(s_{m}, u\right)\right\}$ converges, then $s=u$.

Lemma 2.3 ([10]) Assume that a subset of a complete $C A T(0)$ space $(Y, \theta)$ is closed, convex and $\mathcal{T}: W \rightarrow W$ is a total asymptotically nonexpansive mapping. Let $\left\{s_{m}\right\}$ be a bounded sequence in $W$ such that $\Delta \lim _{m \rightarrow \infty} s_{m}=t$ and $\lim _{m \rightarrow \infty} \theta\left(s_{m}, \mathcal{T} s_{m}\right)=0$. Then $\mathcal{T} t=t$.

Let $\mathcal{C B}(W)$ be a collection of all nonempty and closed bounded subsets and $\mathcal{P}(W)$ be a collection of all nonempty proximal bounded and closed subsets of $W$, respectively. Let $\mathcal{H}(\cdot, \cdot)$ be the Hausdorff distance on $\mathcal{C B}(W)$ defined by

$$
\mathcal{H}(\mathcal{A}, \mathcal{B}):=\max \left\{\sup _{s \in \mathcal{A}} \operatorname{dist}(s, \mathcal{B}), \sup _{r \in \mathcal{B}} \operatorname{dist}(r, \mathcal{A})\right\}, \quad \forall \mathcal{A}, \mathcal{B} \in \mathcal{C B}(W)
$$

A subset $W \subset Y \neq \emptyset$ is said to be proximal if, for each $s \in Y$, there exists an element $r \in W$ such that

$$
\theta(s, r)=\operatorname{dist}(s, W):=\inf \{\theta(s, q): q \in W\}
$$

It is well known that each weakly compact convex subset of a Banach space is proximal as well as each closed convex subset of a uniformly convex Banach space is also proximal.

Let $\mathcal{T}: Y \rightarrow 2^{Y}$ be a multivalued mapping. An element $s \in Y$ is said to be a fixed point of $\mathcal{T}$ if $s \in \mathcal{T} s$.

Definition 2.4 A multivalued mapping $\mathcal{T}: Y \rightarrow C B(Y)$ is called nonexpansive if

$$
\mathcal{H}(\mathcal{T} s, \mathcal{T} r) \leq \theta(\mathcal{T} s, \mathcal{T} r), \quad s, r \in Y
$$

Recall that a function $h: W \rightarrow(-\infty, \infty]$ is said to be convex if, for any geodesic $[s, r]:=$ $\left\{c_{s, r}(a): 0 \leq a \leq 1\right\}:=\{a s \oplus(1-a) r: 0 \leq a \leq 1\}$ joining $s, r \in W$, the function $h \circ c$ is convex, i.e.,

$$
h\left(c_{s, r}(a)\right):=h(a s \oplus(1-a) r) \leq a h(s)+(1-a) h(r) .
$$


For all $\pi>0$, define the Moreau-Yosida resolvent of $h$ in a complete CAT(0) space $Y$ as follows:

$$
\mathcal{J}_{\pi}(s)=\underset{r \in Y}{\arg \min }\left[h(r)+\frac{1}{2 \pi} \theta^{2}(r, s)\right], \quad \forall s \in Y .
$$

Let $h: Y \rightarrow(-\infty, \infty]$ be a proper, convex, and lower semi-continuous function. The set $F\left(\mathcal{J}_{\pi}\right)$ of fixed points of the resolvent $\mathcal{J}_{\pi}$ associated with $h$ coincides with the set $\arg _{r \in Y} \min h(r)$ of minimizers of $h$. Also, for any $\pi>0$, the resolvent $\mathcal{J}_{\pi}$ of $h$ is nonexpansive $[4,12]$.

Lemma 2.4 ([13]) Let $(Y, \theta)$ be a complete $C A T(0)$ space and $h: Y \rightarrow(-\infty, \infty]$ be proper, convex, and lower semi-continuous function. Then, for all $s, r \in Y$ and $\pi>0$, we have

$$
\frac{1}{2 \pi} \theta^{2}\left(\mathcal{J}_{\pi} s, r\right)-\frac{1}{2 \pi} \theta^{2}(s, r)+\frac{1}{2 \pi} \theta^{2}\left(s, \mathcal{J}_{\pi} s\right)+h\left(\mathcal{J}_{\pi} s\right) \leq h(r) .
$$

Lemma $2.5([1])$ Let $(Y, \theta)$ be a complete $C A T(0)$ space and $h: W \rightarrow(-\infty, \infty]$ be a proper convex and lower semi-continuous function. Then the following identity holds:

$$
\mathcal{J}_{\pi} s=\mathcal{J}_{\mu}\left(\frac{\pi-\mu}{\pi} \mathcal{J}_{\pi} s \oplus \frac{\mu}{\pi} s\right), \quad \forall s \in Y
$$

and $\pi>\mu>0$.

Lemma 2.6 ([11]) Let $\left\{a_{m}\right\},\left\{b_{m}\right\}$, and $\left\{r_{m}\right\}$ be sequences of nonnegative real numbers such that

$$
a_{m+1} \leq\left(1+b_{m}\right) a_{m}+r_{m}, \quad \forall m \in \mathbb{N} .
$$

If $\sum_{m=1}^{\infty} b_{m}<\infty$ and $\sum_{m=1}^{\infty} r_{m}<\infty$, then $\lim _{m \rightarrow \infty} a_{m}$ exists.

\section{Convergence results}

Now we construct and prove the main result of this paper.

Theorem 3.1 Let $(Y, \theta)$ be a complete $C A T(0)$ space and $W$ be a nonempty closed convex subset. Let $\mathcal{T}: W \rightarrow \mathcal{P}(W)$ be a multivalued mapping and $\mathcal{P}_{\mathcal{T}}$ be a nonexpansive mapping. Let $h: Y \rightarrow(-\infty, \infty]$ be a proper convex and lower semi-continuous function, $A, B, C: W \rightarrow W$ be three total asymptotically nonexpansive mappings with $\left\{\mu_{m}\right\}$ and $\left\{v_{m}\right\}$ being nonnegative real sequences such that $\sum_{m=1}^{\infty} \mu_{m}<\infty$ and $\sum_{m=1}^{\infty} v_{m}<\infty$. There exists a constant $M_{1}>0$ such that $\zeta(\theta) \leq M_{1} \theta, \theta \geq 0$ with $G(A) \cap G(B) \cap G(C) \neq \emptyset$ and

$$
\Xi:=G(A) \cap G(B) \cap G(C) \cap G(\mathcal{T}) \cap \arg \min _{r \in W} h(r) \neq \emptyset .
$$

Let $\left\{s_{m}\right\}$ be defined by

$$
\left\{\begin{array}{l}
p_{m}=\arg \min _{r \in Y}\left[h(r)+\frac{1}{2 \pi_{m}} \theta^{2}\left(r, s_{m}\right)\right] \\
q_{m}=\left(1-c_{m}\right) p_{m} \oplus c_{m} A^{m} z_{m}, \\
r_{m}=\left(1-b_{m}\right) q_{m} \oplus b_{m} B^{m} y_{m}, \\
s_{m+1}=\left(1-a_{m}\right) r_{m} \oplus a_{m} C^{m} x_{m},
\end{array}\right.
$$


where $z_{m} \in \mathcal{P}_{\mathcal{T}}\left(p_{m}\right), y_{m} \in \mathcal{P}_{\mathcal{T}}\left(q_{m}\right)$, and $x_{m} \in \mathcal{P}_{\mathcal{T}}\left(r_{m}\right)$ for each $m \in \mathbb{N}$. Let $\left\{a_{m}\right\},\left\{b_{m}\right\}$, and $\left\{c_{m}\right\}$ be sequences in $[0,1]$ for all $m \in \mathbb{N}$ and $\left\{\pi_{m}\right\}$ be a sequence with $\pi_{m}>0$ for all $m \in \mathbb{N}$. Then

$$
\lim _{m \rightarrow \infty} \theta\left(s_{m}, t\right) \quad \text { exists for all } t \in \Xi \text {. }
$$

Proof Let $t \in \Xi$. Then $t=$ At $=B t=C t$ and $h(t) \leq h(r)$ for any $r \in W$. So, we have

$$
h(t)+\frac{1}{2 \pi_{m}} \theta^{2}(t, t) \leq h(r)+\frac{1}{2 \pi_{m}} \theta^{2}(r, t)
$$

for each $r \in W$, and so we have $t=\mathcal{J}_{\pi_{m}} t$ for each $m \in \mathbb{N}$. Since $p_{m}=\mathcal{J}_{\pi_{m}} s_{m}$ and $\mathcal{J}_{\pi_{m}}$ is nonexpansive, we have

$$
\theta\left(p_{m}, t\right)=\theta\left(\mathcal{J}_{\pi_{m}} s_{m}, \mathcal{J}_{\pi_{m}} t\right) \leq \theta\left(s_{m}, t\right) .
$$

Now, using (3.1), (3.2), and Lemma 2.1(ii), we have

$$
\begin{aligned}
\theta\left(q_{m}, t\right)= & \theta\left(\left(1-c_{m}\right) p_{m} \oplus c_{m} A^{m} z_{m}, t\right) \\
\leq & \left(1-c_{m}\right) \theta\left(p_{m}, t\right)+c_{m} \theta\left(A^{m} z_{m}, t\right) \\
\leq & \left(1-c_{m}\right) \theta\left(p_{m}, t\right)+c_{m}\left[\theta\left(z_{m}, t\right)+v_{m}\left(\theta\left(z_{m}, t\right)\right)+\mu_{m}\right] \\
\leq & \left(1-c_{m}\right) \theta\left(p_{m}, t\right)+c_{m}\left[\theta\left(z_{m}, t\right)+v_{m} M_{1} \theta\left(z_{m}, t\right)+\mu_{m}\right] \\
= & \left(1-c_{m}\right) \theta\left(p_{m}, t\right)+c_{m}\left(1+v_{m} M_{1}\right) \theta\left(z_{m}, t\right)+c_{m} \mu_{m} \\
\leq & \left(1-c_{m}\right) \operatorname{dist}\left(p_{m}, \mathcal{P}_{\mathcal{T}}(t)\right)+c_{m}\left(1+v_{m} M_{1}\right) \operatorname{dist}\left(z_{m}, \mathcal{P}_{\mathcal{T}}(t)\right)+c_{m} \mu_{m} \\
\leq & \left(1-c_{m}\right) \mathcal{H}\left(\mathcal{P}_{\mathcal{T}}\left(p_{m}\right), \mathcal{P}_{\mathcal{T}}(t)\right)+c_{m}\left(1+v_{m} M_{1}\right) \mathcal{H}\left(\mathcal{P}_{\mathcal{T}}\left(p_{m}\right), \mathcal{P}_{\mathcal{T}}(t)\right) \\
& +c_{m} \mu_{m} \\
\leq & \left(1-c_{m}\right) \theta\left(p_{m}, t\right)+c_{m}\left(1+v_{m} M_{1}\right) \theta\left(p_{m}, t\right)+c_{m} \mu_{m} \\
\leq & \left(1+v_{m} M_{1}\right) \theta\left(p_{m}, t\right)+c_{m} \mu_{m} \\
\leq & \left(1+v_{m} M_{1}\right) \theta\left(s_{m}, t\right)+\mu_{m},
\end{aligned}
$$

and using (3.1), (3.3), and Lemma 2.1(ii), we have

$$
\begin{aligned}
\theta\left(r_{m}, t\right)= & \theta\left(\left(1-b_{m}\right) q_{m} \oplus b_{m} B^{m} y_{m}, t\right) \\
\leq & \left(1-b_{m}\right) \theta\left(q_{m}, t\right)+b_{m} \theta\left(B^{m} y_{m}, t\right) \\
\leq & \left(1-b_{m}\right) \theta\left(q_{m}, t\right)+b_{m}\left[\theta\left(y_{m}, t\right)+v_{m}\left(\theta\left(y_{m}, t\right)\right)+\mu_{m}\right] \\
\leq & \left(1-b_{m}\right) \theta\left(q_{m}, t\right)+b_{m}\left[\theta\left(y_{m}, t\right)+v_{m} M_{1} \theta\left(y_{m}, t\right)+\mu_{m}\right] \\
= & \left(1-b_{m}\right) \theta\left(q_{m}, t\right)+b_{m}\left(1+v_{m} M_{1}\right) \theta\left(y_{m}, t\right)+b_{m} \mu_{m} \\
\leq & \left(1-b_{m}\right) \operatorname{dist}\left(q_{m}, \mathcal{P}_{\mathcal{T}}(t)\right)+b_{m}\left(1+v_{m} M_{1}\right) \operatorname{dist}\left(y_{m}, \mathcal{P}_{\mathcal{T}}(t)\right)+b_{m} \mu_{m} \\
\leq & \left(1-b_{m}\right) \mathcal{H}\left(\mathcal{P}_{\mathcal{T}}\left(q_{m}\right), \mathcal{P}_{\mathcal{T}}(t)\right)+b_{m}\left(1+v_{m} M_{1}\right) \mathcal{H}\left(\mathcal{P}_{\mathcal{T}}\left(q_{m}\right), \mathcal{P}_{\mathcal{T}}(t)\right) \\
& +b_{m} \mu_{m}
\end{aligned}
$$




$$
\begin{aligned}
& \leq\left(1-b_{m}\right) \theta\left(q_{m}, t\right)+b_{m}\left(1+v_{m} M_{1}\right) \theta\left(q_{m}, t\right)+b_{m} \mu_{m} \\
& \leq\left(1+v_{m} M_{1}\right) \theta\left(q_{m}, t\right)+b_{m} \mu_{m} \\
& \leq\left(1+v_{m} M_{1}\right)\left[\left(1+v_{m} M_{1}\right) \theta\left(s_{m}, t\right)+\mu_{m}\right]+b_{m} \mu_{m} \\
& \leq\left(1+v_{m} M_{1}\right)^{2} \theta\left(s_{m}, t\right)+\left(1+v_{m} M_{1}\right) \mu_{m}+b_{m} \mu_{m} \\
& \leq\left(1+v_{m} M_{1}\right)^{2} \theta\left(s_{m}, t\right)+\left(2+v_{m} M_{1}\right) \mu_{m} .
\end{aligned}
$$

Similarly using (3.1), (3.4), and Lemma 2.1(ii), we have

$$
\begin{aligned}
\theta\left(s_{m+1}, t\right)= & \theta\left(\left(1-a_{m}\right) r_{m} \oplus a_{m} C^{m} x_{m}, t\right) \\
\leq & \left(1-a_{m}\right) \theta\left(r_{m}, t\right)+a_{m} \theta\left(C^{m} x_{m}, t\right) \\
\leq & \left(1-a_{m}\right) \theta\left(r_{m}, t\right)+a_{m}\left[\theta\left(x_{m}, t\right)+v_{m}\left(\theta\left(x_{m}, t\right)\right)+\mu_{m}\right] \\
\leq & \left(1-a_{m}\right) \theta\left(r_{m}, t\right)+a_{m}\left[\theta\left(x_{m}, t\right)+v_{m} M_{1} \theta\left(x_{m}, t\right)+\mu_{m}\right] \\
= & \left(1-a_{m}\right) \theta\left(r_{m}, t\right)+a_{m}\left(1+v_{m} M_{1}\right) \theta\left(x_{m}, t\right)+a_{m} \mu_{m} \\
\leq & \left(1-a_{m}\right) \operatorname{dist}\left(r_{m}, \mathcal{P}_{\mathcal{T}}(t)\right)+a_{m}\left(1+v_{m} M_{1}\right) \operatorname{dist}\left(x_{m}, \mathcal{P}_{\mathcal{T}}(t)\right)+a_{m} \mu_{m} \\
\leq & \left(1-a_{m}\right) \mathcal{H}\left(\mathcal{P}_{\mathcal{T}}\left(r_{m}\right), \mathcal{P}_{\mathcal{T}}(t)\right)+a_{m}\left(1+v_{m} M_{1}\right) \mathcal{H}\left(\mathcal{P}_{\mathcal{T}}\left(r_{m}\right), \mathcal{P}_{\mathcal{T}}(t)\right) \\
& +a_{m} \mu_{m} \\
\leq & \left(1-a_{m}\right) \theta\left(r_{m}, t\right)+a_{m}\left(1+v_{m} M_{1}\right) \theta\left(r_{m}, t\right)+a_{m} \mu_{m} \\
\leq & \left(1+v_{m} M_{1}\right) \theta\left(r_{m}, t\right)+a_{m} \mu_{m} \\
\leq & \left(1+v_{m} M_{1}\right)\left[\left(1+v_{m} M_{1}\right)^{2} \theta\left(s_{m}, t\right)+\left(2+v_{m} M_{1}\right) \mu_{m}\right]+a_{m} \mu_{m} \\
\leq & \left(1+v_{m} M_{1}\right)^{3} \theta\left(s_{m}, t\right)+\left(1+v_{m} M_{1}\right)\left(2+v_{m} M_{1}\right) \mu_{m}+a_{m} \mu_{m} \\
\leq & \left(1+v_{m} M_{1}\right)^{3} \theta\left(s_{m}, t\right)+3\left(1+v_{m} M_{1}\right) \mu_{m} \\
= & \left(1+\mathcal{S}_{m}\right) \theta\left(s_{m}, t\right)+\mathcal{W}_{m},
\end{aligned}
$$

where $\mathcal{S}_{m}=3 M_{1} v_{m}+3 M_{1}^{2} v_{m}^{2}+M_{1}^{3} v_{m}^{3}$ and $\mathcal{W}_{m}=3\left(1+v_{m} M_{1}\right) \mu_{m}$. Since it is given that $\sum_{m=1}^{\infty} \mu_{m}<\infty$ and $\sum_{m=1}^{\infty} v_{m}<\infty$, we get $\sum_{m=1}^{\infty} \mathcal{S}_{m}<\infty$ and $\sum_{m=1}^{\infty} \mathcal{W}_{m}<\infty$. From Lemma 2.6, (3.5) we have $\lim _{m \rightarrow \infty} \theta\left(s_{m}, t\right)$ exists, and we assume that

$$
\lim _{m \rightarrow \infty} \theta\left(s_{m}, t\right)=\kappa \geq 0
$$

From (3.6), $\left\{s_{m}\right\}$ is bounded and so the sequences $\left\{p_{m}\right\},\left\{q_{m}\right\},\left\{r_{m}\right\},\left\{A^{m} s_{m}\right\},\left\{B^{m} s_{m}\right\}$, and $\left\{C^{m} s_{m}\right\}$ are bounded.

Theorem 3.2 Let $(Y, \theta)$ be a complete $C A T(0)$ space and $W$ be a nonempty closed convex subset. Let $\mathcal{T}: W \rightarrow \mathcal{P}(W)$ be a multivalued mapping and $\mathcal{P}_{\mathcal{T}}$ be a nonexpansive mapping. Let $h: Y \rightarrow(-\infty, \infty]$ be a proper convex and lower semi-continuous function and $A, B, C: W \rightarrow W$ be three total asymptotically nonexpansive mappings with $\left\{\mu_{m}\right\}$ and $\left\{v_{m}\right\}$ being nonnegative real sequences such that $\sum_{m=1}^{\infty} \mu_{m}<\infty$ and $\sum_{m=1}^{\infty} v_{m}<\infty$. There exists a constant $M_{1}>0$ such that $\zeta(\theta) \leq M_{1} \theta, \theta \geq 0$, with $G(A) \cap G(B) \cap G(C) \neq \emptyset$ and

$$
\Xi:=G(A) \cap G(B) \cap G(C) \cap G(\mathcal{T}) \cap \arg \min _{r \in W} h(r) \neq \emptyset
$$


Let $\left\{s_{m}\right\}$ be defined by

$$
\left\{\begin{array}{l}
p_{m}=\arg \min _{r \in Y}\left[h(r)+\frac{1}{2 \pi_{m}} \theta^{2}\left(r, s_{m}\right)\right] \\
q_{m}=\left(1-c_{m}\right) p_{m} \oplus c_{m} A^{m} z_{m}, \\
r_{m}=\left(1-b_{m}\right) q_{m} \oplus b_{m} B^{m} y_{m}, \\
s_{m+1}=\left(1-a_{m}\right) r_{m} \oplus a_{m} C^{m} x_{m},
\end{array}\right.
$$

where $z_{m} \in \mathcal{P}_{\mathcal{T}}\left(p_{m}\right), y_{m} \in \mathcal{P}_{\mathcal{T}}\left(q_{m}\right)$, and $x_{m} \in \mathcal{P}_{\mathcal{T}}\left(r_{m}\right)$ for each $m \in \mathbb{N}$. Let $\left\{a_{m}\right\},\left\{b_{m}\right\}$, and $\left\{c_{m}\right\}$ be sequences in $[0,1]$ for all $m \in \mathbb{N}$ and $\left\{\pi_{m}\right\}$ be a sequence with $\pi_{m}>0$ for all $m \in \mathbb{N}$.

Then

(i) $\lim _{m \rightarrow \infty} \theta\left(s_{m}, p_{m}\right)=0$;

(ii) $\lim _{m \rightarrow \infty} \theta\left(s_{m}, A s_{m}\right)=\lim _{m \rightarrow \infty} \theta\left(s_{m}, B s_{m}\right)=\lim _{m \rightarrow \infty} \theta\left(s_{m}, C s_{m}\right)=0$.

Proof (i) By Lemma 2.4, we have

$$
\frac{1}{2 \pi_{m}}\left\{\theta^{2}\left(p_{m}, t\right)-\theta^{2}\left(s_{m}, t\right)+\theta^{2}\left(s_{m}, p_{m}\right)\right\} \leq h(t)-h\left(p_{m}\right) .
$$

Since $h(t) \leq h\left(p_{m}\right)$ for each $m \in \mathbb{N}$, we have

$$
\theta^{2}\left(s_{m}, p_{m}\right) \leq \theta^{2}\left(s_{m}, t\right)-\theta^{2}\left(p_{m}, t\right) .
$$

From (3.5), we have

$$
\theta\left(s_{m+1}, t\right) \leq\left(1+v_{m} M_{1}\right) \theta\left(r_{m}, t\right)+a_{m} \mu_{m}
$$

and

$$
\lim _{m \rightarrow \infty} \inf \theta\left(r_{m}, t\right) \geq \kappa .
$$

From (3.4), we have

$$
\lim _{m \rightarrow \infty} \sup \theta\left(r_{m}, t\right) \leq \kappa .
$$

So, from (3.8) and (3.9), we have

$$
\lim _{m \rightarrow \infty} \theta\left(r_{m}, t\right)=\kappa .
$$

Similarly, from (3.4), we have

$$
\theta\left(r_{m}, t\right) \leq\left(1+v_{m} M_{1}\right) \theta\left(q_{m}, t\right)+b_{m} \mu_{m}
$$

and

$$
\lim _{m \rightarrow \infty} \inf \theta\left(q_{m}, t\right) \geq \kappa
$$


From (3.3), we have

$$
\lim _{m \rightarrow \infty} \sup \theta\left(q_{m}, t\right) \leq \kappa
$$

From (3.11) and (3.12), we have

$$
\lim _{m \rightarrow \infty} \theta\left(q_{m}, t\right)=\kappa
$$

From (3.3), we have

$$
\theta\left(q_{m}, t\right) \leq\left(1+v_{m} M_{1}\right) \theta\left(p_{m}, t\right)+c_{m} \mu_{m}
$$

and

$$
\lim _{m \rightarrow \infty} \inf \theta\left(p_{m}, t\right) \geq \kappa
$$

Also from (3.3), we have

$$
\lim _{m \rightarrow \infty} \sup \theta\left(p_{m}, t\right) \leq \kappa .
$$

From (3.14) and (3.15), we have

$$
\lim _{m \rightarrow \infty} \theta\left(p_{m}, t\right)=\kappa
$$

So from (3.7), we have

$$
\lim _{m \rightarrow \infty} \theta\left(s_{m}, p_{m}\right)=0
$$

(ii) Suppose that $\Xi$ is nonempty, and let $t \in \Xi$. From (3.6), $\lim _{m \rightarrow \infty} \theta\left(s_{m}, t\right)$ exists and $\left\{s_{m}\right\}$ is bounded. From (3.1) and Lemma 2.1(i), we have

$$
\begin{aligned}
\theta^{2}\left(q_{m}, t\right)= & \theta^{2}\left(\left(1-c_{m}\right) p_{m} \oplus c_{m} A^{m} z_{m}, t\right) \\
\leq & \left(1-c_{m}\right) \theta^{2}\left(p_{m}, t\right)+c_{m} \theta^{2}\left(A^{m} z_{m}, t\right)-c_{m}\left(1-c_{m}\right) \theta^{2}\left(p_{m}, A^{m} z_{m}\right) \\
\leq & \left(1-c_{m}\right) \theta^{2}\left(p_{m}, t\right)+c_{m}\left[\theta\left(z_{m}, t\right)+v_{m}\left(\theta\left(z_{m}, t\right)\right)+\mu_{m}\right]^{2} \\
& -c_{m}\left(1-c_{m}\right) \theta^{2}\left(p_{m}, A^{m} z_{m}\right) \\
\leq & c_{m}\left[\theta\left(z_{m}, t\right)+v_{m} M_{1} \theta\left(z_{m}, t\right)+\mu_{m}\right]^{2}+\left(1-c_{m}\right) \theta^{2}\left(p_{m}, t\right) \\
& -c_{m}\left(1-c_{m}\right) \theta^{2}\left(A^{m} z_{m}, p_{m}\right) \\
= & c_{m}\left[\left(1+v_{m} M_{1}\right) \theta\left(z_{m}, t\right)+\mu_{m}\right]^{2}+\left(1-c_{m}\right) \theta^{2}\left(p_{m}, t\right) \\
& -c_{m}\left(1-c_{m}\right) \theta^{2}\left(A^{m} z_{m}, p_{m}\right) \\
\leq & \left(1+v_{m} M_{1}\right)^{2} c_{m} \theta^{2}\left(z_{m}, t\right)+\left(1+v_{m} M_{1}\right)^{2}\left(1-c_{m}\right) \theta^{2}\left(p_{m}, t\right) \\
& +c_{m}\left[2\left(1+v_{m} M_{1}\right) \mu_{m} \theta\left(z_{m}, t\right)+\mu_{m}^{2}\right]-c_{m}\left(1-c_{m}\right) \theta^{2}\left(A^{m} z_{m}, p_{m}\right) \\
\leq & \left(1+v_{m} M_{1}\right)^{2} c_{m} \operatorname{dist}\left(z_{m}, \mathcal{P}_{\mathcal{T}}(t)\right)^{2}+\left(1+v_{m} M_{1}\right)^{2}\left(1-c_{m}\right) \operatorname{dist}\left(p_{m}, \mathcal{P}_{\mathcal{T}}(t)\right)^{2}
\end{aligned}
$$




$$
\begin{aligned}
& +c_{m}\left[2\left(1+v_{m} M_{1}\right) \mu_{m} \operatorname{dist}\left(z_{m}, \mathcal{P}_{T}(t)\right)+\mu_{m}^{2}\right]-c_{m}\left(1-c_{m}\right) \theta^{2}\left(p_{m}, A^{m} z_{m}\right) \\
\leq & \left(1+v_{m} M_{1}\right)^{2} c_{m} \mathcal{H}\left(\mathcal{P}_{\mathcal{T}}\left(p_{m}\right), \mathcal{P}_{\mathcal{T}}(t)\right)^{2} \\
& +\left(1+v_{m} M_{1}\right)^{2}\left(1-c_{m}\right) \mathcal{H}\left(\mathcal{P}_{\mathcal{T}}\left(p_{m}\right), \mathcal{P}_{\mathcal{T}}(t)\right)^{2} \\
& +c_{m}\left[2\left(1+v_{m} M_{1}\right) \mu_{m} \mathcal{H}\left(\mathcal{P}_{\mathcal{T}}\left(p_{m}\right), \mathcal{P}_{T}(t)\right)+\mu_{m}^{2}\right]-c_{m}\left(1-c_{m}\right) \theta^{2}\left(p_{m}, A^{m} z_{m}\right) \\
\leq & \left(1+v_{m} M_{1}\right)^{2} c_{m} \theta^{2}\left(p_{m}, t\right)+\left(1+v_{m} M_{1}\right)^{2}\left(1-c_{m}\right) \theta^{2}\left(p_{m}, t\right) \\
& +c_{m}\left[2\left(1+v_{m} M_{1}\right) \mu_{m} \theta\left(p_{m}, t\right)+\mu_{m}^{2}\right]-c_{m}\left(1-c_{m}\right) \theta^{2}\left(p_{m}, A^{m} z_{m}\right) \\
= & \left(1+v_{m} M_{1}\right)^{2} \theta^{2}\left(p_{m}, t\right)+c_{m}\left[2\left(1+v_{m} M_{1}\right) \mu_{m} \theta\left(p_{m}, t\right)+\mu_{m}^{2}\right] \\
& -c_{m}\left(1-c_{m}\right) \theta^{2}\left(p_{m}, A^{m} z_{m}\right) \\
\leq & \left(1+v_{m} M_{1}\right)^{2} \theta^{2}\left(s_{m}, t\right)+c_{m}\left[2\left(1+v_{m} M_{1}\right) \mu_{m} \theta\left(s_{m}, t\right)+\mu_{m}^{2}\right] \\
& -c_{m}\left(1-c_{m}\right) \theta^{2}\left(p_{m}, A^{m} z_{m}\right) \\
\leq & \theta^{2}\left(s_{m}, t\right)+p v_{m}+q \mu_{m}-c_{m}\left(1-c_{m}\right) \theta^{2}\left(p_{m}, A^{m} z_{m}\right)
\end{aligned}
$$

for some $p, q>0$, which implies that

$$
\theta^{2}\left(q_{m}, t\right) \leq \theta^{2}\left(s_{m}, t\right)+p v_{m}+q \mu_{m}
$$

and from (3.1) and Lemma 2.1(i), we have

$$
\begin{aligned}
\theta^{2}\left(r_{m}, t\right)= & \theta^{2}\left(\left(1-b_{m}\right) q_{m} \oplus b_{m} B^{m} y_{m}, t\right) \\
\leq & \left(1-b_{m}\right) \theta^{2}\left(q_{m}, t\right)+b_{m} \theta^{2}\left(B^{m} y_{m}, t\right)-b_{m}\left(1-b_{m}\right) \theta^{2}\left(q_{m}, B^{m} y_{m}\right) \\
\leq & \left(1-b_{m}\right) \theta^{2}\left(q_{m}, t\right)+b_{m}\left[\theta\left(y_{m}, t\right)+v_{m}\left(\theta\left(y_{m}, t\right)\right)+\mu_{m}\right]^{2} \\
& -b_{m}\left(1-b_{m}\right) \theta^{2}\left(q_{m}, B^{m} y_{m}\right) \\
\leq & \left(1-b_{m}\right) \theta^{2}\left(q_{m}, t\right)+b_{m}\left[\theta\left(y_{m}, t\right)+v_{m} M_{1} \theta\left(y_{m}, t\right)+\mu_{m}\right]^{2} \\
& -b_{m}\left(1-b_{m}\right) \theta^{2}\left(B^{m} y_{m}, q_{m}\right) \\
= & \left(1-b_{m}\right) \theta^{2}\left(q_{m}, t\right)+b_{m}\left[\left(1+v_{m} M_{1}\right) \theta\left(y_{m}, t\right)+\mu_{m}\right]^{2} \\
& -b_{m}\left(1-b_{m}\right) \theta^{2}\left(B^{m} y_{m}, q_{m}\right) \\
\leq & \left(1-b_{m}\right)\left(1+v_{m} M_{1}\right)^{2} \theta^{2}\left(q_{m}, t\right)+\left(1+v_{m} M_{1}\right)^{2} b_{m} \theta^{2}\left(y_{m}, t\right) \\
& +b_{m}\left[2\left(1+v_{m} M_{1}\right) \mu_{m} \theta\left(y_{m}, t\right)+\mu_{m}^{2}\right]-b_{m}\left(1-b_{m}\right) \theta^{2}\left(B^{m} y_{m}, q_{m}\right) \\
\leq & \left(1-b_{m}\right)\left(1+v_{m} M_{1}\right)^{2} \operatorname{dist}\left(q_{m}, \mathcal{P}_{\mathcal{T}}(t)\right)^{2}+\left(1+v_{m} M_{1}\right)^{2} b_{m} \operatorname{dist}\left(y_{m}, \mathcal{P}_{\mathcal{T}}(t)\right)^{2} \\
& +b_{m}\left[2\left(1+v_{m} M_{1}\right) \mu_{m} \operatorname{dist}\left(y_{m}, \mathcal{P}_{\mathcal{T}}(t)\right)+\mu_{m}^{2}\right]-b_{m}\left(1-b_{m}\right) \theta^{2}\left(B^{m} y_{m}, q_{m}\right) \\
\leq & \left(1-b_{m}\right)\left(1+v_{m} M_{1}\right)^{2} \mathcal{H}\left(\mathcal{P}_{\mathcal{T}}\left(q_{m}\right), \mathcal{P}_{\mathcal{T}}(t)\right)^{2} \\
& +\left(1+v_{m} M_{1}\right)^{2} b_{m} \mathcal{H}\left(\mathcal{P}_{\mathcal{T}}\left(q_{m}\right), \mathcal{P}_{\mathcal{T}}(t)\right)^{2} \\
& +b_{m}\left[2\left(1+v_{m} M_{1}\right) \mu_{m} \mathcal{H}\left(\mathcal{P}_{\mathcal{T}}\left(q_{m}\right), \mathcal{P}_{\mathcal{T}}(t)\right)+\mu_{m}^{2}\right]-b_{m}\left(1-b_{m}\right) \theta^{2}\left(B^{m} y_{m}, q_{m}\right) \\
\leq & \left(1-b_{m}\right)\left(1+v_{m} M_{1}\right)^{2} \theta^{2}\left(q_{m}, t\right)+\left(1+v_{m} M_{1}\right)^{2} b_{m} \theta^{2}\left(q_{m}, t\right) \\
& +b_{m}\left[2\left(1+v_{m} M_{1}\right) \mu_{m} \theta\left(q_{m}, t\right)+\mu_{m}^{2}\right]-b_{m}\left(1-b_{m}\right) \theta^{2}\left(B^{m} y_{m}, q_{m}\right) \\
&
\end{aligned}
$$




$$
\begin{aligned}
= & \left(1+v_{m} M_{1}\right)^{2} \theta^{2}\left(q_{m}, t\right)+b_{m}\left[2\left(1+v_{m} M_{1}\right) \mu_{m} \theta\left(q_{m}, t\right)+\mu_{m}^{2}\right] \\
& -b_{m}\left(1-b_{m}\right) \theta^{2}\left(B^{m} y_{m}, q_{m}\right) \\
\leq & \theta^{2}\left(q_{m}, t\right)+r v_{m}+s \mu_{m}-b_{m}\left(1-b_{m}\right) \theta^{2}\left(B^{m} y_{m}, q_{m}\right)
\end{aligned}
$$

for some $r, s>0$, which implies that

$$
\theta^{2}\left(r_{m}, t\right) \leq \theta^{2}\left(q_{m}, t\right)+r v_{m}+s \mu_{m} .
$$

Similarly, from (3.1) and Lemma 2.1(i), we have

$$
\begin{aligned}
\theta^{2}\left(s_{m+1}, t\right)= & \theta^{2}\left(\left(1-a_{m}\right) r_{m} \oplus a_{m} C^{m} x_{m}, t\right) \\
\leq & \left(1-a_{m}\right) \theta^{2}\left(r_{m}, t\right)+a_{m} \theta^{2}\left(C^{m} x_{m}, t\right)-a_{m}\left(1-a_{m}\right) \theta^{2}\left(r_{m}, C^{m} x_{m}\right) \\
\leq & \left(1-a_{m}\right) \theta^{2}\left(r_{m}, t\right)+a_{m}\left[\theta\left(x_{m}, t\right)+v_{m}\left(\theta\left(x_{m}, t\right)\right)+\mu_{m}\right]^{2} \\
& -a_{m}\left(1-a_{m}\right) \theta^{2}\left(r_{m}, C^{m} x_{m}\right) \\
\leq & \left(1-a_{m}\right) \theta^{2}\left(r_{m}, t\right)+a_{m}\left[\theta\left(x_{m}, t\right)+v_{m} M_{1} \theta\left(x_{m}, t\right)+\mu_{m}\right]^{2} \\
& -a_{m}\left(1-a_{m}\right) \theta^{2}\left(C^{m} x_{m}, r_{m}\right) \\
= & \left(1-a_{m}\right) \theta^{2}\left(r_{m}, t\right)+a_{m}\left[\left(1+v_{m} M_{1}\right) \theta\left(x_{m}, t\right)+\mu_{m}\right]^{2} \\
& -a_{m}\left(1-a_{m}\right) \theta^{2}\left(C^{m} x_{m}, r_{m}\right) \\
\leq & \left(1-a_{m}\right)\left(1+v_{m} M_{1}\right)^{2} \theta^{2}\left(r_{m}, t\right)+\left(1+v_{m} M_{1}\right)^{2} a_{m} \theta^{2}\left(x_{m}, t\right) \\
& +a_{m}\left[2\left(1+v_{m} M_{1}\right) \mu_{m} \theta\left(x_{m}, t\right)+\mu_{m}^{2}\right]-a_{m}\left(1-a_{m}\right) \theta^{2}\left(C^{m} x_{m}, r_{m}\right) \\
\leq & \left(1-a_{m}\right)\left(1+v_{m} M_{1}\right)^{2} \operatorname{dist}\left(r_{m}, \mathcal{P}_{\mathcal{T}}(t)\right)^{2}+\left(1+v_{m} M_{1}\right)^{2} a_{m} \operatorname{dist}\left(x_{m}, \mathcal{P}_{\mathcal{T}}(t)\right)^{2} \\
& +a_{m}\left[2\left(1+v_{m} M_{1}\right) \mu_{m} \operatorname{dist}\left(x_{m}, \mathcal{P}_{\mathcal{T}}(t)\right)+\mu_{m}^{2}\right]-a_{m}\left(1-a_{m}\right) \theta^{2}\left(C^{m} x_{m}, r_{m}\right) \\
\leq & \left(1-a_{m}\right)\left(1+v_{m} M_{1}\right)^{2} \mathcal{H}\left(\mathcal{P}_{\mathcal{T}}\left(r_{m}\right), \mathcal{P}_{\mathcal{T}}(t)\right)^{2}+\left(1+v_{m} M_{1}\right)^{2} a_{m} \\
& \times \\
& \mathcal{H}\left(\mathcal{P}_{\mathcal{T}}\left(r_{m}\right), \mathcal{P}_{\mathcal{T}}(t)\right)^{2}+a_{m}\left[2\left(1+v_{m} M_{1}\right) \mu_{m} \mathcal{H}\left(\mathcal{P}_{\mathcal{T}}\left(r_{m}\right), \mathcal{P}_{\mathcal{T}}(t)\right)+\mu_{m}^{2}\right] \\
& -a_{m}\left(1-a_{m}\right) \theta^{2}\left(C^{m} x_{m}, r_{m}\right) \\
\leq & \left(1-a_{m}\right)\left(1+v_{m} M_{1}\right)^{2} \theta^{2}\left(r_{m}, t\right)+\left(1+v_{m} M_{1}\right)^{2} a_{m} \theta^{2}\left(r_{m}, t\right) \\
& +a_{m}\left[2\left(1+v_{m} M_{1}\right) \mu_{m} \theta\left(r_{m}, t\right)+\mu_{m}^{2}\right]-a_{m}\left(1-a_{m}\right) \theta^{2}\left(C^{m} x_{m}, r_{m}\right) \\
= & \left(1+v_{m} M_{1}\right)^{2} \theta^{2}\left(r_{m}, t\right)+a_{m}\left[2\left(1+v_{m} M_{1}\right) \mu_{m} \theta\left(r_{m}, t\right)+\mu_{m}^{2}\right] \\
& -a_{m}\left(1-a_{m}\right) \theta^{2}\left(C^{m} x_{m}, r_{m}\right) \\
\leq & \theta^{2}\left(r_{m}, t\right)+t v_{m}+w \mu_{m}-a_{m}\left(1-a_{m}\right) \theta^{2}\left(C^{m} x_{m}, r_{m}\right) \\
& \\
&
\end{aligned}
$$

for some $t, w>0$, which implies that

$$
\theta^{2}\left(s_{m+1}, t\right) \leq \theta^{2}\left(r_{m}, t\right)+t v_{m}+w \mu_{m}
$$

From (3.18), we have

$$
c_{m}\left(1-c_{m}\right) \theta^{2}\left(A^{m} z_{m}, p_{m}\right) \leq \theta^{2}\left(s_{m}, t\right)-\theta^{2}\left(q_{m}, t\right)+p v_{m}+q \mu_{m} .
$$


Since $\sum_{m=1}^{\infty} v_{m}<\infty, \sum_{m=1}^{\infty} \mu_{m}<\infty$, we have

$$
c_{m}\left(1-c_{m}\right) \theta^{2}\left(A^{m} z_{m}, p_{m}\right)<\infty
$$

From $\liminf _{m \rightarrow \infty} c_{m}\left(1-c_{m}\right)>0$, we have

$$
\lim _{m \rightarrow \infty} \theta\left(A^{m} z_{m}, p_{m}\right)=0
$$

From (3.19), we have

$$
b_{m}\left(1-b_{m}\right) \theta^{2}\left(B^{m} y_{m}, q_{m}\right) \leq \theta^{2}\left(q_{m}, t\right)-\theta^{2}\left(r_{m}, t\right)+r v_{m}+s \mu_{m}
$$

Since $\sum_{m=1}^{\infty} v_{m}<\infty, \sum_{m=1}^{\infty} \mu_{m}<\infty$, we have

$$
b_{m}\left(1-b_{m}\right) \theta^{2}\left(B^{m} y_{m}, q_{m}\right)<\infty \text {. }
$$

From $\liminf _{m \rightarrow \infty} b_{m}\left(1-b_{m}\right)>0$, we have

$$
\lim _{m \rightarrow \infty} \theta\left(B^{m} y_{m}, q_{m}\right)=0
$$

From (3.20), we have

$$
a_{m}\left(1-a_{m}\right) \theta^{2}\left(C^{m} x_{m}, r_{m}\right) \leq \theta^{2}\left(r_{m}, t\right)-\theta^{2}\left(s_{m+1}, t\right)+t v_{m}+w \mu_{m} .
$$

Since $\sum_{m=1}^{\infty} v_{m}<\infty, \sum_{m=1}^{\infty} \mu_{m}<\infty$, we have

$$
a_{m}\left(1-a_{m}\right) \theta^{2}\left(C^{m} x_{m}, r_{m}\right)<\infty
$$

From $\liminf _{m \rightarrow \infty} a_{m}\left(1-a_{m}\right)>0$, we have

$$
\lim _{m \rightarrow \infty} \theta\left(C^{m} x_{m}, r_{m}\right)=0
$$

Now using (3.17) and (3.21), we have

$$
\begin{aligned}
\theta\left(q_{m}, s_{m}\right) & =\theta\left(\left(1-c_{m}\right) p_{m} \oplus c_{m} A^{m} z_{m}, s_{m}\right) \\
& \leq\left(1-c_{m}\right) \theta\left(p_{m}, s_{m}\right)+c_{m} \theta\left(A^{m} z_{m}, s_{m}\right) \\
& \leq\left(1-c_{m}\right) \theta\left(p_{m}, s_{m}\right)+c_{m}\left[\theta\left(A^{m} z_{m}, p_{m}\right)+\theta\left(p_{m}, s_{m}\right)\right] \\
& \leq\left(1-c_{m}\right) \theta\left(p_{m}, s_{m}\right)+c_{m} \theta\left(A^{m} z_{m}, p_{m}\right)+c_{m} \theta\left(p_{m}, s_{m}\right) \\
& =\theta\left(p_{m}, s_{m}\right)+c_{m} \theta\left(A^{m} z_{m}, p_{m}\right) \\
& \rightarrow 0 \quad \text { as } m \rightarrow \infty,
\end{aligned}
$$

and using (3.22) and (3.24), we have

$$
\theta\left(r_{m}, s_{m}\right)=\theta\left(\left(1-b_{m}\right) q_{m} \oplus b_{m} B^{m} y_{m}, s_{m}\right)
$$




$$
\begin{aligned}
& \leq\left(1-b_{m}\right) \theta\left(q_{m}, s_{m}\right)+b_{m} \theta\left(B^{m} y_{m}, s_{m}\right) \\
& \leq\left(1-b_{m}\right) \theta\left(q_{m}, s_{m}\right)+b_{m}\left[\theta\left(B^{m} y_{m}, q_{m}\right)+\theta\left(q_{m}, s_{m}\right)\right] \\
& \leq\left(1-b_{m}\right) \theta\left(q_{m}, s_{m}\right)+b_{m} \theta\left(B^{m} y_{m}, q_{m}\right)+b_{m} \theta\left(q_{m}, s_{m}\right) \\
& =\theta\left(q_{m}, s_{m}\right)+b_{m} \theta\left(B^{m} y_{m}, q_{m}\right) \\
& \rightarrow 0 \quad \text { as } m \rightarrow \infty,
\end{aligned}
$$

and again using (3.23) and (3.25), we have

$$
\begin{aligned}
\theta\left(s_{m+1}, s_{m}\right) & =\theta\left(\left(1-a_{m}\right) r_{m} \oplus a_{m} C^{m} x_{m}, s_{m}\right) \\
& \leq\left(1-a_{m}\right) \theta\left(r_{m}, s_{m}\right)+a_{m} \theta\left(C^{m} x_{m}, s_{m}\right) \\
& \leq\left(1-a_{m}\right) \theta\left(r_{m}, s_{m}\right)+a_{m}\left[\theta\left(C^{m} x_{m}, r_{m}\right)+\theta\left(r_{m}, s_{m}\right)\right] \\
& \leq\left(1-a_{m}\right) \theta\left(r_{m}, s_{m}\right)+a_{m} \theta\left(C^{m} x_{m}, r_{m}\right)+a_{m} \theta\left(r_{m}, s_{m}\right) \\
& =\theta\left(r_{m}, s_{m}\right)+a_{m} \theta\left(C^{m} x_{m}, r_{m}\right) \\
& \rightarrow 0 \quad \text { as } m \rightarrow \infty .
\end{aligned}
$$

Using the triangle inequality (3.17) and (3.21), we have

$$
\begin{aligned}
\theta\left(A^{m} s_{m}, s_{m}\right) & \leq \theta\left(A^{m} s_{m}, A^{m} z_{m}\right)+\theta\left(A^{m} z_{m}, p_{m}\right)+\theta\left(p_{m}, s_{m}\right) \\
& \leq L \theta\left(s_{m}, z_{m}\right)+\theta\left(A^{m} z_{m}, p_{m}\right)+\theta\left(p_{m}, s_{m}\right) \\
& =L \mathcal{H}\left(\mathcal{P}_{\mathcal{T}}\left(s_{m}\right), \mathcal{P}_{\mathcal{T}}\left(p_{m}\right)\right)+\theta\left(A^{m} z_{m}, p_{m}\right)+\theta\left(p_{m}, s_{m}\right) \\
& \leq L \theta\left(s_{m}, p_{m}\right)+\theta\left(A^{m} z_{m}, p_{m}\right)+\theta\left(p_{m}, s_{m}\right) \\
& \leq(L+1) \theta\left(s_{m}, p_{m}\right)+\theta\left(A^{m} z_{m}, p_{m}\right) \\
& \rightarrow 0 \quad \text { as } m \rightarrow \infty .
\end{aligned}
$$

Again using the triangle inequality (3.22) and (3.24), we have

$$
\begin{aligned}
\theta\left(B^{m} s_{m}, s_{m}\right) & \leq \theta\left(B^{m} s_{m}, B^{m} y_{m}\right)+\theta\left(B^{m} y_{m}, q_{m}\right)+\theta\left(q_{m}, s_{m}\right) \\
& \leq L \theta\left(s_{m}, y_{m}\right)+\theta\left(B^{m} y_{m}, q_{m}\right)+\theta\left(q_{m}, s_{m}\right) \\
& =L \mathcal{H}\left(\mathcal{P}_{\mathcal{T}}\left(s_{m}\right), \mathcal{P}_{\mathcal{T}}\left(q_{m}\right)\right)+\theta\left(B^{m} y_{m}, q_{m}\right)+\theta\left(q_{m}, s_{m}\right) \\
& \leq L \theta\left(s_{m}, q_{m}\right)+\theta\left(B^{m} y_{m}, q_{m}\right)+\theta\left(q_{m}, s_{m}\right) \\
& \leq(L+1) \theta\left(s_{m}, q_{m}\right)+\theta\left(B^{m} y_{m}, q_{m}\right) \\
& \rightarrow 0 \quad \text { as } m \rightarrow \infty .
\end{aligned}
$$

Similarly, again using the triangle inequality (3.23) and (3.25), we have

$$
\begin{aligned}
\theta\left(C^{m} s_{m}, s_{m}\right) & \leq \theta\left(C^{m} s_{m}, C^{m} x_{m}\right)+\theta\left(C^{m} x_{m}, r_{m}\right)+\theta\left(r_{m}, s_{m}\right) \\
& \leq L \theta\left(s_{m}, x_{m}\right)+\theta\left(C^{m} x_{m}, r_{m}\right)+\theta\left(r_{m}, s_{m}\right)
\end{aligned}
$$




$$
\begin{aligned}
& =L \mathcal{H}\left(\mathcal{P}_{\mathcal{T}}\left(s_{m}\right), \mathcal{P}_{\mathcal{T}}\left(r_{m}\right)\right)+\theta\left(C^{m} x_{m}, r_{m}\right)+\theta\left(r_{m}, s_{m}\right) \\
& \leq L \theta\left(s_{m}, r_{m}\right)+\theta\left(C^{m} x_{m}, r_{m}\right)+\theta\left(r_{m}, s_{m}\right) \\
& \leq(L+1) \theta\left(s_{m}, r_{m}\right)+\theta\left(C^{m} x_{m}, r_{m}\right) \\
& \rightarrow 0 \quad \text { as } m \rightarrow \infty .
\end{aligned}
$$

Now, using (3.26) and (3.27), we have

$$
\begin{aligned}
\theta\left(s_{m}, A s_{m}\right) \leq & \theta\left(s_{m}, s_{m+1}\right)+\theta\left(s_{m+1}, A^{m+1} s_{m+1}\right)+\theta\left(A^{m+1} s_{m+1}, A^{m+1} s_{m}\right) \\
& +\theta\left(A^{m+1} s_{m}, A s_{m}\right) \\
\leq & \theta\left(s_{m}, s_{m+1}\right)+\theta\left(s_{m+1}, A^{m+1} s_{m+1}\right)+L \theta\left(s_{m+1}, s_{m}\right)+L \theta\left(A^{m} s_{m}, s_{m}\right) \\
\rightarrow & 0 \quad \text { as } m \rightarrow \infty .
\end{aligned}
$$

Similarly, we have

$$
\lim _{m \rightarrow \infty} \theta\left(s_{m}, B s_{m}\right)=\lim _{m \rightarrow \infty} \theta\left(s_{m}, C s_{m}\right)=0 .
$$

Theorem 3.3 Let $W$ be a nonempty closed convex subset of a complete CAT(0) space. Let $\mathcal{T}: W \rightarrow \mathcal{P}(W)$ be a multivalued mapping and $\mathcal{P}_{\mathcal{T}}$ be a nonexpansive mapping. Let $h$ : $Y \rightarrow(-\infty, \infty]$ be a proper convex and lower semi-continuous function and $A, B, C: W \rightarrow$ $W$ be three total asymptotically nonexpansive mappings. Then $\left\{s_{m}\right\}$ given by (3.1) is $\Delta$ convergent to a common fixed point of $\Xi$.

Proof From Lemma 2.5 and (3.17), we have

$$
\begin{aligned}
\theta\left(\mathcal{J}_{\pi} s_{m}, s_{m}\right) & \leq \theta\left(\mathcal{J}_{\pi} s_{m}, p_{m}\right)+\theta\left(p_{m}, s_{m}\right) \\
& =\theta\left(\mathcal{J}_{\pi} s_{m}, \mathcal{J}_{\pi_{m}} s_{m}\right)+\theta\left(p_{m}, s_{m}\right) \\
& =\theta\left(\mathcal{J}_{\pi} s_{m}, \mathcal{J}_{\pi}\left(\frac{\pi_{m}-\pi}{\pi_{m}} \mathcal{J}_{\pi_{m}} s_{m} \oplus \frac{\pi}{\pi_{m}} s_{m}\right)\right)+\theta\left(p_{m}, s_{m}\right) \\
& \leq \theta\left(s_{m},\left(1-\frac{\pi}{\pi_{m}}\right) \mathcal{J}_{\pi_{m}} s_{m} \oplus \frac{\pi}{\pi_{m}} s_{m}\right)+\theta\left(p_{m}, s_{m}\right) \\
& \leq\left(1-\frac{\pi}{\pi_{m}}\right) \theta\left(s_{m}, \mathcal{J}_{\pi_{m}} s_{m}\right)+\frac{\pi}{\pi_{m}} \theta\left(s_{m}, s_{m}\right)+\theta\left(p_{m}, s_{m}\right) \\
& =\left(1-\frac{\pi}{\pi_{m}}\right) \theta\left(s_{m}, p_{m}\right)+\theta\left(p_{m}, s_{m}\right) \\
& \rightarrow 0 \quad \text { as } m \rightarrow \infty .
\end{aligned}
$$

From Theorem 3.1, we have $\lim _{m \rightarrow \infty} \theta\left(s_{m}, t\right)$ exists for all $t \in \Xi$ and

$$
\lim _{m \rightarrow \infty} \theta\left(s_{m}, A s_{m}\right)=\lim _{m \rightarrow \infty} \theta\left(s_{m}, B s_{m}\right)=\lim _{m \rightarrow \infty} \theta\left(s_{m}, C s_{m}\right)=0 .
$$

Next, we have to show that

$$
\mathcal{W}_{\Delta}\left(s_{m}\right)=\bigcup_{\left\{u_{m}\right\} \subset\left\{s_{m}\right\}} \mathcal{A}\left(\left\{u_{n}\right\}\right) \subset \Xi .
$$


Let $u \in \mathcal{W}_{\Delta}\left(s_{m}\right)$. Then there exists a subsequence $\left\{u_{m}\right\}$ of $\left\{s_{m}\right\}$ such that $\mathcal{A}\left(\left\{u_{m}\right\}\right)=\{u\}$. From Definition 2.3, there exists a subsequence $\left\{v_{m}\right\}$ of $\left\{u_{m}\right\}$ such that $\Delta-\lim _{m \rightarrow \infty} v_{m}=v$ for some $v \in W$. By Lemma 2.3, $v \in \Xi$. By Lemma 2.2, $u=v$. This shows that $\mathcal{W}_{\Delta}\left(s_{m}\right) \subset \Xi$.

Finally, we have to prove that the sequence $\left\{s_{m}\right\} \Delta$-converges to a point in $\Xi$, which will prove that $\mathcal{W}_{\Delta}\left(s_{m}\right)$ consists of exactly one point. Let $\left\{u_{m}\right\}$ be a subsequence of $\left\{s_{m}\right\}$ with $\mathcal{A}\left(\left\{u_{m}\right\}\right)=\{u\}$, and let $\mathcal{A}\left(\left\{s_{m}\right\}\right)=\{s\}$. Since $u \in \mathcal{W}_{\Delta}\left(s_{m}\right) \subset \Xi$ and $\left\{\theta\left(s_{m}, u\right)\right\}$ converges by Lemma 2.2, we have $s=u$. Thus, $\mathcal{W}_{\Delta}\left(s_{m}\right)=\{s\}$.

Corollary 3.1 Let $W$ be a nonempty closed convex subset of a real Hilbert space $H$. Let $\mathcal{T}: W \rightarrow \mathcal{P}(W)$ be a multivalued mapping, let $h: Y \rightarrow(\infty, \infty]$ be a proper convex and lower semi-continuous function, and $A, B, C, z_{m} \in \mathcal{P}_{\mathcal{T}}\left(p_{m}\right), y_{m} \in \mathcal{P}_{\mathcal{T}}\left(q_{m}\right), x_{m} \in$ $\mathcal{P}_{\mathcal{T}}\left(r_{m}\right),\left\{a_{m}\right\},\left\{b_{m}\right\},\left\{c_{m}\right\}$, and $\left\{\pi_{m}\right\}$ satisfy all the conditions given in Theorem 3.1. Let $\left\{s_{m}\right\}$ be the sequence given by

$$
\left\{\begin{array}{l}
p_{m}=\arg \min _{r \in Y}\left[h(r)+\frac{1}{2 \pi_{m}}\left\|r-s_{m}\right\|^{2}\right] \\
q_{m}=\left(1-c_{m}\right) p_{m} \oplus c_{m} A^{m} z_{m} \\
r_{m}=\left(1-b_{m}\right) q_{m} \oplus b_{m} B^{m} y_{m} \\
s_{m+1}=\left(1-a_{m}\right) r_{m} \oplus a_{m} C^{m} x_{m}
\end{array}\right.
$$

for each $m \in \mathbb{N}$, then the sequence $\left\{s_{m}\right\}$ converges weakly to a common point in $\Xi$.

Now we construct and prove strong convergence theorems.

Let $W$ be a nonempty closed convex subset of a $\operatorname{CAT}(0)$ space $(Y, \theta)$. A family $\{A, B, C, \mathcal{T}\}$ of mappings is said to satisfy Condition $(\Xi)$ if there exists a nondecreasing function $h$ : $[0, \infty) \rightarrow[0, \infty)$ with $h(0)=0$ and $h(w)>0$ for all $w \in(0, \infty)$ such that

$$
\theta(s, A s) \geq h(\theta(s, \mathcal{G}))
$$

or

$$
\theta(s, B s) \geq h(\theta(s, \mathcal{G}))
$$

or

$$
\theta(s, C s) \geq h(\theta(s, \mathcal{G}))
$$

or

$$
\theta(s, \mathcal{T} s) \geq h(\theta(s, \mathcal{G}))
$$

for all $s \in Y$, where $\mathcal{G}=G(A) \cap G(B) \cap G(C) \cap G(\mathcal{T})$.

Theorem 3.4 Suppose that the conditions in Theorem 3.1 are given and $\left\{A, B, C, \mathcal{J}_{\pi}\right\}$ satisfy Condition $(\Xi)$. Then the sequence $\left\{s_{m}\right\}$ strongly converges to a common element of $\Xi$. 
Proof From Theorem 3.1 we have $\lim _{m \rightarrow \infty} \theta\left(s_{m}, t\right)$ exists for all $t \in \Xi$. Also it follows that $\lim _{m \rightarrow \infty} \theta\left(s_{m}, \Xi\right)$ exists. On the other hand, by Condition $\Xi$, we have

$$
\lim _{m \rightarrow \infty} \theta\left(\theta\left(s_{m}, \Xi\right)\right) \geq \lim _{m \rightarrow \infty} \theta\left(s_{m}, A s_{m}\right)=0,
$$

or

$$
\lim _{m \rightarrow \infty} \theta\left(\theta\left(s_{m}, \Xi\right)\right) \geq \lim _{m \rightarrow \infty} \theta\left(s_{m}, B s_{m}\right)=0
$$

or

$$
\lim _{m \rightarrow \infty} \theta\left(\theta\left(s_{m}, \Xi\right)\right) \geq \lim _{m \rightarrow \infty} \theta\left(s_{m}, C s_{m}\right)=0,
$$

or

$$
\lim _{m \rightarrow \infty} \theta\left(\theta\left(s_{m}, \Xi\right)\right) \geq \lim _{m \rightarrow \infty} \theta\left(s_{m}, \mathcal{J}_{\pi} s_{m}\right)=0 .
$$

Thus, we have $\lim _{m \rightarrow \infty} h\left(\theta\left(s_{m}, \Xi\right)\right)=0$. By using the property of $h$, we have

$$
\lim _{m \rightarrow \infty} \theta\left(s_{m}, \Xi\right)=0
$$

Thus, $\left\{s_{m}\right\}$ is a Cauchy sequence in $Y$, and so $\left\{s_{m}\right\}$ converges to a point $t \in Y$ and hence $\theta(t, \Xi)=0$. Since $\Xi$ is closed, so we have $t \in \Xi$.

Remark 3.1 Our results extend the results of Cho et al. [14] in the framework of CAT(0) spaces. They established convergence theorems for three asymptotically quasinonexpansive mappings involving the convex and lower semi-continuous function for solving the convex minimization problem and the common fixed point problem.

\section{Numerical results}

In this section, we give a numerical example to illustrate the convergence of the iterative algorithm given by (3.1) for supporting our main results.

Example 4.1 Let $Y=\mathbb{R}$ be a Euclidean metric space and $W=[1,12]$. Let $\mathcal{T}: W \rightarrow \mathcal{P}(W)$ be the mapping defined by

$$
\mathcal{T}(s)=\{2 s-1\}, \quad \forall s \in W .
$$

Suppose that $A, B, C: \mathbb{R} \rightarrow \mathbb{R}$ are the mappings defined by

$$
A(s)=\frac{5 s+1}{6} ; \quad B(s)=\sqrt{2 s^{2}-2 s+1} \quad \text { and } \quad C(s)=\sqrt[3]{s^{2}+s-1} .
$$

For all $s \in W$, let $h: Y \rightarrow(-\infty, \infty]$ defined by

$$
h(s)=\|s\|_{1}+\frac{1}{2}\|s\|_{2}^{2}-2 s+1 .
$$


Table 1 Numerical values of $s_{m}$ and $\left\|s_{m}-s_{m-1}\right\|_{2}$

\begin{tabular}{llll}
\hline No. of iterations & $s_{m}$ & $h\left(s_{m}\right)$ & $\left\|s_{m}-s_{m-1}\right\|_{2}$ \\
\hline$m=1$ & 10 & 51 & - \\
$m=2$ & 2.887912 & 2.267415 & 5.143740 \\
$m=3$ & 1.513123 & 0.6300537 & 4.117401 \\
$m=4$ & 1.230808 & 0.528876 & 1.801738 \\
$m=5$ & 1.153017 & 0.511232 & 0.583212 \\
$m=6$ & 1.119232 & 0.507252 & 0.120032 \\
$m=7$ & 1.098545 & 0.504013 & 0.000651 \\
$m=8$ & 1.083867 & 0.503242 & $2.680507 \times \mathrm{e}^{-2}$ \\
$m=9$ & 1.063823 & 0.502737 & $3.324120 \times \mathrm{e}^{-4}$ \\
$m=10$ & 1.056611 & 0.502344 & $9.929354 \times \mathrm{e}^{-7}$ \\
$\mathrm{~m}=11$ & 1.041421 & 0.500856 & $2.906031 \times \mathrm{e}^{-9}$ \\
$\mathrm{~m}=12$ & 1.028531 & 0.500547 & $1.170353 \times \mathrm{e}^{-11}$ \\
$\mathrm{~m}=13$ & 1.017221 & 0.500236 & $2.224861 \times \mathrm{e}^{-14}$ \\
$\mathrm{~m}=14$ & 1.016117 & 0.500228 & $5.773151 \times \mathrm{e}^{-19}$ \\
$\mathrm{~m}=15$ & 1.009912 & 0.500179 & 0.00000 \\
$\mathrm{~m}=16$ & 1.009234 & 0.500132 & 0.00000 \\
$\mathrm{~m}=17$ & 1.008221 & 0.500101 & 0.00000 \\
$\mathrm{~m}=18$ & 1.007608 & 0.500097 & 0.00000 \\
$\mathrm{~m}=19$ & 1.006803 & 0.500082 & 0.00000 \\
$\mathrm{~m}=20$ & 1.004818 & 0.500060 & 0.00000 \\
$\mathrm{~m}=21$ & 1.003001 & 0.500012 & 0.00000 \\
$\mathrm{~m}=22$ & 1.000387 & 0.500001 & 0.00000 \\
$\mathrm{~m}=23$ & 1.000201 & 0.500000 & 0.00000 \\
$\mathrm{~m}=24$ & 1.000000 & 0.500000 & 0.00000 \\
$\mathrm{~m}=25$ & 1.000000 & 0.500000 & 0.00000 \\
\hline
\end{tabular}

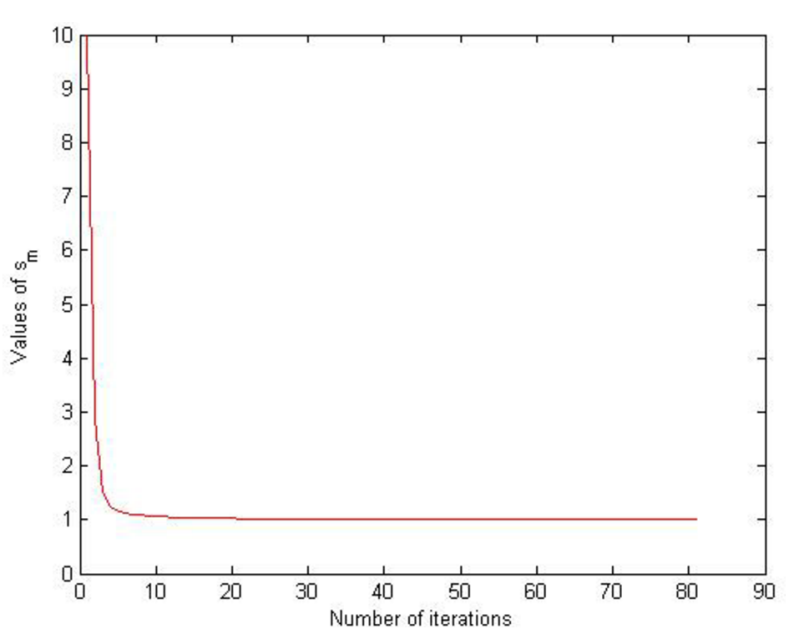

Figure 1 The values of $h\left(s_{m}\right)$ given in Table 1

It is easy to check that $A, B, C$ are uniformly continuous and total asymptotically nonexpansive mappings with $G(A) \cap G(B) \cap G(C) \cap G(\mathcal{T})=\{1\}$ and $h$ is a proper convex and lower semi-continuous function.

Let $a_{m}=\frac{3 m-2}{6 m}, b_{m}=\frac{7 m-3}{11 m}$, and $c_{m}=\frac{11 m-7}{19 m}$, and $s_{1}=10$ is the initial value. Then we obtain numerical results with the error values in Table 1.

From Table 1 and Fig. 1, we see that the sequence $\left\{s_{m}\right\}$ converges to 1 which is a common fixed point of three asymptotically nonexpansive mappings, multivalued mapping, and a solution of a minimizer of a function $h$. 


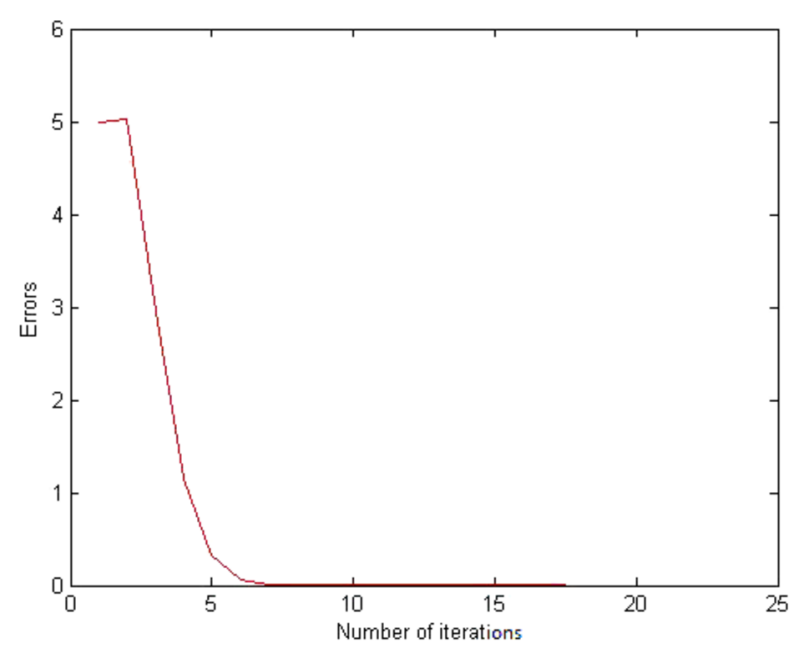

Figure 2 The values of errors given in Table 1

\section{Conclusion}

In the above section, we prove the $\Delta$-convergence of a modified proximal point algorithm for common fixed points in a CAT(0) space for generalized nonexpansive mappings which includes a total asymptotically nonexpansive mapping, a multivalued mapping, and a minimizer of a convex function. Moreover, we have illustrated our result by a numerical example which supports the $\Delta$-convergence of our proposed algorithm.

Acknowledgements

Not applicable.

Funding

Not applicable.

Abbreviations

Not applicable.

Availability of data and materials

Data sharing not applicable to this paper as no datasets were generated or analyzed during the current study.

Competing interests

The authors declare that they have no competing interests.

Authors' contributions

Both authors contributed equally and significantly in writing this paper. Both authors read and approved the final manuscript.

\section{Publisher's Note}

Springer Nature remains neutral with regard to jurisdictional claims in published maps and institutional affiliations.

Received: 6 October 2018 Accepted: 18 April 2019 Published online: 08 May 2019

\section{References}

1. Martinet, B.: Regularisation dineuations variationnelles par approximations successives. Rev. Fr. Inform. Rech. Oper. 4 , 154-158 (1970)

2. Rockafellar, R.T.: Monotone operators and the proximal point algorithm. SIAM J. Control Optim. 14, $877-898$ (1976)

3. Bacak, M.: The proximal point algorithm in metric spaces. Isr. J. Math. 194, 689-701 (2013)

4. Ariza-Ruiz, D., Leustean, L., Lopez, G.: Firmly nonexpansive mappings in classes of geodesic spaces. Trans. Am. Math. Soc. 366, 4299-4322 (2014)

5. Cholamjiak, P.: The modified proximal point algorithm in CAT(0) spaces. Optim. Lett. 9, 1401-1410 (2015)

6. Cholamjiak, P., Abdou, A.A., Cho, Y.J.: Proximal point algorithms involving fixed points of nonexpansive mappings in CAT(0) spaces. Fixed Point Theory Appl. 2015, 227 (2015) 
7. Cheng, S.S., Yao, J.C., Wang, L., Qin, L.J.: Some convergence theorems involving proximal point and common fixed points for asymptotically nonexpansive mappings in CAT(0) spaces. Fixed Point Theory Appl. 2016, 68 (2016)

8. Kitkuan, D., Padcharoen, A.: Strong convergence of a modified SP-iteration process for generalized asymptotically quasi-nonexpansive mappings in CAT(0) spaces. J. Nonlinear Sci. Appl. 9, 2126-2135 (2016)

9. Markin, J.T.: Continuous dependence of fixed point sets. Proc. Am. Math. Soc. 38, 545-547 (1973)

10. Nadler, S.B.: Multivalued contraction mappings. Pac. J. Math. 30, 475-488 (1969)

11. Shimizu, T., Takahashi, W.: Fixed points of multivalued mappings in certain convex metric spaces. Topol. Methods Nonlinear Anal. 8, 197-203 (1996)

12. Chang, S.S., Wang, L., Lee, H.W.J., Chan, C.K., Yang, L.: Demiclosed principle and $\Delta$-convergence theorems for total asymptotically nonexpansive mappings in CAT(0) spaces. Appl. Math. Comput. 219, 2611-2617 (2012)

13. Ambrosio, L., Gigli, N., Savare, G.: Gradient Flows in Metric Spaces and in the Space of Probability Measures. Lectures in Mathematics ETH Zurich. Birkhäuser, Basel (2008)

14. Pakkaranang, N., Kumam, P., Cho, Y.J.: Proximal point algorithms for solving convex minimization problem and common fixed points problem of asymptotically quasi-nonexpansive mappings in CAT(0) spaces with convergence analysis. Numer. Algorithms 78(3), 827-845 (2018)

Submit your manuscript to a SpringerOpen ${ }^{\circ}$ journal and benefit from:

- Convenient online submission

- Rigorous peer review

- Open access: articles freely available online

- High visibility within the field

- Retaining the copyright to your article

Submit your next manuscript at $\boldsymbol{\nabla}$ springeropen.com 